\title{
Acute Inflammatory Bowel Disease Complicating Chronic Alcoholism and Mimicking Carcinoid Syndrome
}

\author{
Piercarlo Ballo $^{a}$ Pietro Dattolo $^{b}$ Giuseppe Mangialavori $^{a}$ \\ Giuseppe Ferro $^{b}$ Francesca Fusco $^{c}$ Matteo Consalvo ${ }^{c}$ \\ Leandro Chiodi $^{\mathrm{a}}$ Francesco Pizzarelli $^{\mathrm{b}} \quad$ Alfredo Zuppiroli $^{\mathrm{d}}$ \\ ${ }^{a}$ Cardiology Unit, ${ }^{b}$ Nephrology and Dialysis Unit and ${ }^{c}$ Radiology Unit, \\ S. Maria Annunziata Hospital, and ${ }^{\mathrm{d} D e p a r t m e n t}$ of Cardiology, Local Health \\ Unit, Florence, Italy
}

\section{Key Words}

Acute inflammatory bowel disease $\cdot$ Chronic alcoholism $\cdot$ Carcinoid syndrome

\begin{abstract}
We report the case of a woman with a history of chronic alcohol abuse who was hospitalized with diarrhea, severe hypokalemia refractory to potassium infusion, nausea, vomiting, abdominal pain, alternations of high blood pressure with phases of hypotension, irritability and increased urinary 5-hydroxyindoleacetic acid and cortisol. Although carcinoid syndrome was hypothesized, abdominal computed tomography and colonoscopy showed non-specific inflammatory bowel disease with severe colic wall thickening, and multiple colic biopsies confirmed non-specific inflammation with no evidence of carcinoid cells. During the following days diarrhea slowly decreased and the patient's condition progressively improved. One year after stopping alcohol consumption, the patient was asymptomatic and serum potassium was normal. Chronic alcohol exposure is known to have several deleterious effects on the intestinal mucosa and can favor and sustain local inflammation. Chronic alcohol intake may also be associated with high blood pressure, behavior disorders, abnormalities in blood pressure regulation with episodes of hypotension during hospitalization due to impaired baroreflex sensitivity in the context of an alcohol withdrawal syndrome, increased urinary 5-hydroxyindoleacetic acid as a result of malabsorption syndrome, and increased urinary cortisol as a result of hypothalamic-pituitary-adrenal axis dysregulation. These considerations, together with the regression of symptoms and normalization of potassium levels after stopping alcohol consumption, suggest the intriguing possibility of a alcohol-related acute inflammatory bowel disease mimicking carcinoid syndrome.
\end{abstract}




\section{Case Report}

A 65-year-old woman with a history of chronic alcohol abuse, obesity, and recent traumatic lumbar fracture was transferred from a rehabilitation center to our hospital because of refractory diarrhea with severe hypokalemia $(1.7 \mathrm{mEq} / \mathrm{l})$. Her therapy included folic acid, sulpiride, ramipril, spironolactone, potassium chloride, delorazepam and nadroparin. Physical examination at admission showed poor general condition, spatiotemporal disorientation, drowsiness and irritability. Blood pressure was $160 / 100 \mathrm{~mm} \mathrm{Hg}$, heart rate was $96 \mathrm{bpm}$, and there were no abnormalities in chest and cardiac examination. Electrocardiography showed sinus tachycardia, and no significant abnormalities were found at echocardiography. Arterial blood gas analysis showed severe metabolic alkalosis. Laboratory investigations showed microcytic anemia ( $\mathrm{Hb} 9.8 \mathrm{~g} / \mathrm{dl}$, MCV $63.5 \mathrm{fl}, \mathrm{MCH} 20.7 \mathrm{pg}$ ), hypoproteinemia $(4.4 \mathrm{~g} / \mathrm{dl})$ and increased serum levels of gamma-GT (94 IU/l), ammonia (71 $\mu \mathrm{g} / \mathrm{dl})$, NT-proBNP $(1,151 \mathrm{pg} / \mathrm{ml})$ and C-reactive protein $(2.58 \mathrm{mg} / \mathrm{dl})$. Because of the increased arrhythmic risk related to severe potassium depletion, the patient was transferred to the intensive care unit.

Parenteral nutrition and treatment with rifaximin and high-dose intravenous potassium (up to $160 \mathrm{mEq}$ /day) were started. Microbiologic tests on stool, including detection of Clostridium difficile and its toxin, were negative. Extensive tumoral, infectiologic, endocrinologic and rheumatologic blood and urinary investigations yielded all normal results, except for an increase in urinary 5-hydroxyindoleacetic acid (12.3 mg/24 h, normal value $<9.0 \mathrm{mg} / 24 \mathrm{~h})$, urinary cortisol $(810 \mu \mathrm{g} / 24 \mathrm{~h}$, normal value $<100 \mu \mathrm{g} / 24 \mathrm{~h})$ and serum CA125 (150 IU/ml, normal value $<35 \mathrm{IU} / \mathrm{ml}$ ). Despite treatment, diarrhea and severe hypokalemia persisted during the following days. On day 6 from admission, the potassium level was still $2.2 \mathrm{mEq} / \mathrm{l}$. Nausea, vomiting, abdominal pain and alternations of high blood pressure (up to $167 / 104 \mathrm{~mm} \mathrm{Hg}$ ) with phases of hypotension (up to $85 / 50 \mathrm{~mm} \mathrm{Hg}$ ) were also observed. Carcinoid syndrome was suspected. Abdominal ultrasound examination showed inhomogeneous hepatic architecture with no other significant abnormalities. Abdominal multi-detector computed tomography, performed before and after contrast intravenous media administration with double arterial and portal phase, showed diffuse inflammation of the entire colon with severe wall thickening and mural stratification, suggesting not-specific inflammatory bowel disease [1] (fig. 1). No involvement of the small bowel was found, and there were no findings suggestive of carcinoid tumor or specific inflammatory bowel diseases, inclu ding Comb sign with prominent vasa recta, mesenteric fat stranding, extramural lymph nodes or mucosal ulcers. Colonoscopy also showed diffuse edema and hyperemia of the colic mucosa with no evidence of ulcerative lesions, and multiple colic biopsies confirmed non-specific inflammatory bowel disease with no evidence of carcinoid cells. Indium-111 OctreoScan was planned, but the patient refused further examinations. During the following days, diarrhea slowly decreased and the patient's condition progressively improved. On day 21, the potassium level was $2.7 \mathrm{mEq} / \mathrm{l}$. The patient was transferred to a long-term care facility, and an educational program aimed at stopping alcohol consumption was planned. Potassium levels returned within normal range after several weeks. At one-year follow-up after stopping alcohol intake, the patient's general condition was good. She had no longer shown diarrhea and her potassium level was $4.5 \mathrm{mEq} / \mathrm{l}$.

\section{Discussion}

This report describes the case of an alcoholic woman who developed severe hypokalemia secondary to refractory diarrhea with instrumental evidence of marked inflammation of the colic wall, suggesting the intriguing possibility of alcohol-related acute inflammatory bowel disease. To our knowledge, there are no previous reports of inflammatory bowel disease associated with alcoholism. Chronic alcohol exposure is known to have a number of deleterious effect on the intestinal mucosa, which depend on complex interaction between increased oxidative stress, hyperpermeability, alcoholic neuropathy and dysbiosis, which finally favors and sustains local inflammation [2,3]. Alcohol may also act as a trigger for flare in patients in whom inflammatory bowel disease has already developed [4]. On the other hand, carcinoid syndrome - an array of symptoms and signs occurring in $10 \%$ of patients with 
carcinoid tumors, related to hyperincretion of serotonin by neoplastic neuroendocrine cells - is characterized by variable combinations of symptoms that may include refractory diarrhea, flushing, electrolyte disturbances, nausea, vomiting, abdominal cramps, hypertensive or hypotensive crisis, wheezing and sometimes right-sided heart failure. Behavior disorders such as irritability and lack of impulse control are also relatively common in patients with carcinoid syndrome, as a consequence of abnormalities in the central serotoninergic system [5]. Notably, diarrhea and flushing the two most typical symptoms of the syndrome - are both present only in $58 \%$ of cases, and may be both absent in up to $22 \%$ of cases $[6,7]$. The diagnosis is based on clinical picture and typical laboratory findings such as increased urinary excretion of 5-hydroxyindoleacetic acid, imaging examinations including standard echography, computed tomography and scintigraphic techniques, and detection of neuroendocrine tumor cells in intestinal biopsies. Laboratory investigations may also show increased urinary cortisol and raised levels of tumor markers including CA125 [8, 9].

Interestingly, the clinical and laboratory presentation in our patient resembled that of carcinoid syndrome. The patient presented with refractory diarrhea, severe hypokalemia, abdominal pain, alternation of phases of high blood pressure and hypotension, and behavior disorders with impulse control abnormalities. Laboratory tests showed increased urinary levels of 5-hydroxyindoleacetic acid, urinary cortisol, and serum CA125. These findings were all consistent with the hypothesis of carcinoid syndrome. However, multiple bowel biopsies did not confirm this diagnosis. Although the impossibility of performing a scintigraphic imaging test in our patient cannot allow to definitely rule out the presence of true carcinoid syndrome, it should be considered that diarrhea, hypokalemia and abdominal pain may also be caused by chronic alcohol consumption via its deleterious effects on the intestinal mucosa. Chronic alcohol intake is also typically associated with high blood pressure [10], and abnormalities in blood pressure regulation with episodes of hypotension are commonly observed in alcoholics during hospitalization as a result of impaired baroreflex sensitivity in the context of alcohol withdrawal syndrome [11]. Behavior disorders with impulse control abnormalities may be favored by chronic alcoholism through its effects on the central nervous system $[12,13]$. Moreover, as a result of malabsorption syndrome, chronic alcohol exposure is often associated with increased urinary excretion of tryptophan-serotonin metabolites such as 5-hydroxyindoleacetic acid [14], whereas alcohol-related hypothalamic-pituitary-adrenal axis dysregulation may lead to an increase in urinary cortisol [15]. Finally, the improvement in symptoms and normalization of potassium level at one-year follow-up after stopping alcohol consumption in our patient also supports the hypothesis of alcohol-related acute inflammatory bowel disease.

In conclusion, this report suggests that chronic alcohol abuse may lead to acute reversible inflammatory bowel disease, refractory diarrhea and severe hypokalemia, with clinical and laboratory features that can mimic those of carcinoid syndrome.

\section{Disclosure Statement}

The authors declare no conflicts of interest. 


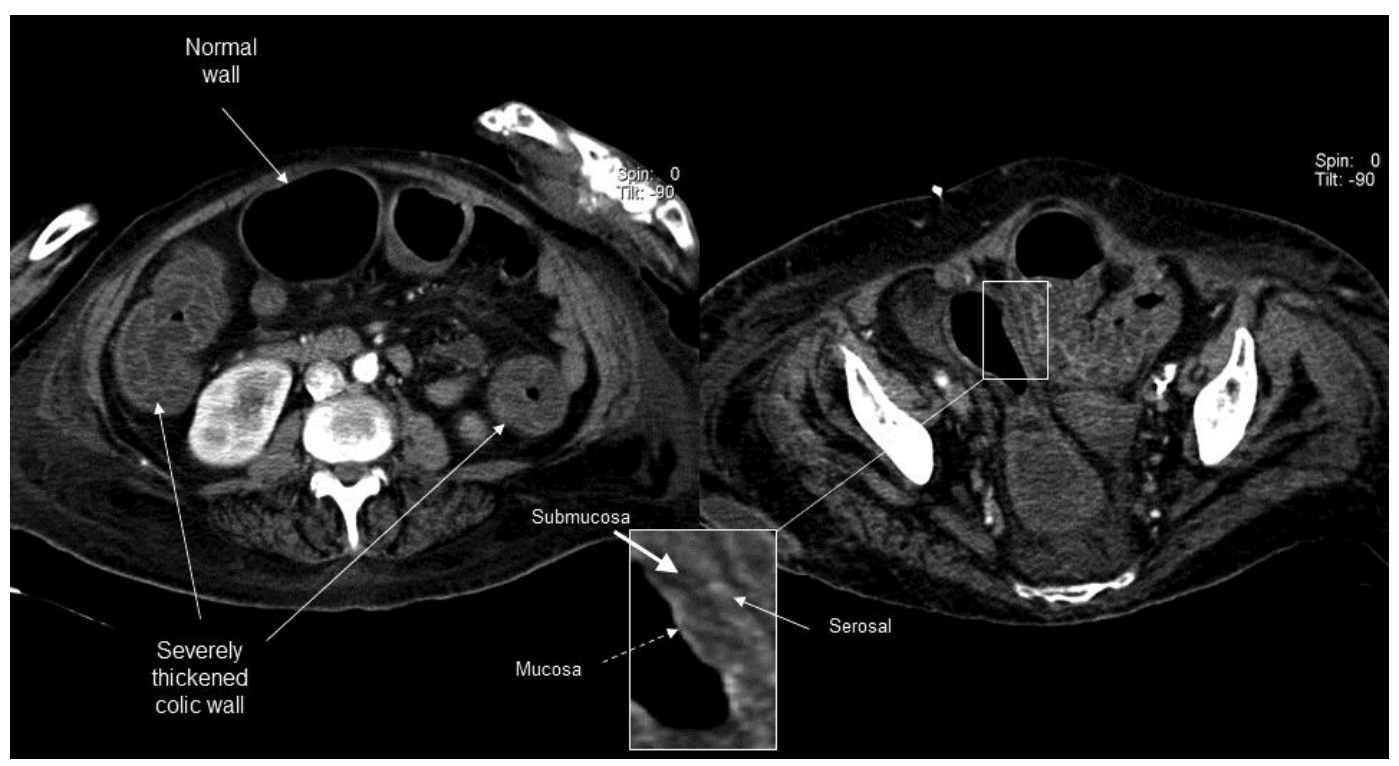

Fig. 1. Computed tomography images showing severe colic inflammation. A marked colic wall thickening with trilaminar appearance was found, with enhanced outer serosal, mucosal hyperemia and attenuation of the interposed submucosal layer due to acute parietal edema. These findings were suggestive of non-specific acute inflammatory bowel disease.

\section{References}

1 Elsayes KM, Al-Hawary MM, Jagdish J, Ganesh HS, Platt JF: CT enterography: principles, trends, and interpretation of findings. Radiographics 2010;30:1955-1970.

-2 Keshavarzian A, Farhadi A, Forsyth CB, Rangan J, Jakate S, Shaikh M, Banan A, Fields JZ: Evidence that chronic alcohol exposure promotes intestinal oxidative stress, intestinal hyperpermeability and endotoxemia prior to development of alcoholic steatohepatitis in rats. J Hepatol 2009;50:538-547.

-3 Mutlu E, Keshavarzian A, Engen P, Forsyth CB, Sikaroodi M, Gillevet P: Intestinal dysbiosis: a possible mechanism of alcohol-induced endotoxemia and alcoholic steatohepatitis in rats. Alcohol Clin Exp Res 2009;33:1836-1846.

-4 Swanson GR, Sedghi S, Farhadi A, Keshavarzian A: Pattern of alcohol consumption and its effect on gastrointestinal symptoms in inflammatory bowel disease. Alcohol 2010;44:223-228.

5 Russo S, Boon JC, Kema IP, Willemse PH, den Boer JA, Korf J, de Vries EG: Patients with carcinoid syndrome exhibit symptoms of aggressive impulse dysregulation. Psychosom Med 2004;66:422-425.

6 Vinik AI, Feliberti E, Perry R, Nakave A: Diffuse hormonal systems and endocrine tumor syndromes. 2008. http://www.endotext.org/guthormones/index.htm.

-7 Van der Lely AJ, de Herder WW: Carcinoid syndrome: diagnosis and medical management. Arq Bras Endocrinol Metabol 2005;49:850-860.

8 Tanke MA, Kema IP, Dijck-Brouwer J, Doornbos B, De Vries EG, Korf J: Low plasma tryptophan in carcinoid patients is associated with increased urinary cortisol excretion. Psychoneuroendocrinology 2008;33:1297-1301.

-9 Portale TR, Mosca F, Minona E, Trovato MA, Gangemi P, Bordonaro R, Puleo S: Gastrointestinal carcinoid tumor and chylous ascites, a rare association with a poor prognosis. A case report. Tumori 2008;94: 419-421.

10 Marmot MG, Elliott P, Shipley MJ, Dyer AR, Ueshima H, Beevers DG, Stamler R, Kesteloot H, Rose G, Stamler J: Alcohol and blood pressure: the INTERSALT study. BMJ 1994;308:1263-1267.

-11 Bär KJ, Boettger MK, Boettger S, Grotelüschen M, Neubauer R, Jochum T, Baier V, Sauer H, Voss A: Reduced baroreflex sensitivity in acute alcohol withdrawal syndrome and in abstained alcoholics. Drug Alcohol Depend 2006;85:66-74. 
12 Rogers RD, Moeller FG, Swann AC, Clark L: Recent research on impulsivity in individuals with drug use and mental health disorders: implications for alcoholism. Alcohol Clin Exp Res 2010;34:1319-1333.

-13 Schellekens AF, de Bruijn ER, van Lankveld CA, Hulstijn W, Buitelaar JK, de Jong CA, Verkes RJ: Alcohol dependence and anxiety increase error-related brain activity. Addiction 2010;105:1928-1934.

14 Das SK, Dhanya L, Vasudevan DM: Biomarkers of alcoholism: an updated review. Scand J Clin Lab Invest 2008;68:81-92.

15 Thayer JF, Hall M, Sollers JJ 3rd, Fischer JE: Alcohol use, urinary cortisol, and heart rate variability in apparently healthy men: evidence for impaired inhibitory control of the HPA axis in heavy drinkers. Int J Psychophysiol 2006;59:244-250. 